\title{
Task-specific strategies of mental "rotation" of facial representations
}

\author{
EDWARD L. COCHRAN \\ Adelphi University, Garden City, New York 11530 \\ and \\ ANNE D. PICK and HERBERT L. PICK, JR. \\ University of Minnesota, Minneapolis, Minnesota 55455
}

\begin{abstract}
The effects of task demands on the visual comparison of facial patterns and of comparable nonfacial patterns were explored in two studies. The studies yielded two primary findings. First, faces, despite their holistic properties, are not rotated faster than comparable non-facelike patterns, although subjects' judgments of them were uniformly more rapid than judgments for nonfaces. Second, the nature of the same-different judgment task required of subjects had a large effect on the pattern of results obtained: When stimuli were compared to their mirror images, results indicative of mental "rotation" were obtained. When stimuli were compared on the basis of similarity of individual features, the pattern of results was very different. This one manipulation produced effects that exceeded those of all of the other manipulations, including that of rotation.
\end{abstract}

There has been considerable interest over the past several years in the manipulation of mental representations. In particular, work by Shepard and his colleagues on mental rotation (e.g., Cooper \& Shepard, 1973) has rekindled controversy about the nature of mental representations and of the processes by which they are transformed. Shepard and others (e.g., Kosslyn, 1981; Kosslyn \& Pomerantz, 1977; Shepard, 1978) have described an analog model of representation: There exists a one-to-one correspondence (at some level of analysis) between points on a real object and points of the mental representation of that object. This correspondence is maintained during mental transformation as if the real object were itself being physically transformed (Shepard, 1978, p. 134). Hence, mental representations are holistic; they are manipulated in ways analogous, or isomorphic, to those in which physical objects are manipulated. They can be scanned, rotated, expanded, and so on.

Others (e.g., Palmer, 1975; Pylyshyn, 1973, 1978, 1979,1981 ) have taken issue with the analog model for various reasons and, instead, argue that some form of a propositional structure is better able to account for

Portions of this report were presented at the annual meeting of the Midwestern Psychological Association, St. Louis, 1980. This research was supported by grants to the University of Minnesota Center for Research in Human Learning from the National Science Foundation and the National Institutes of Child Health and Human Development (HD 01136, HD 00098, and HD 07151) and grants to the Institu te of Child Development from the National Institutes of Child Health and Human Development (HD 05027). Reprint requests may be sent to the first author, at the Department of Psychology, Adelphi University, Garden City, New York 11530. the evidence about mental representation. In this view, the representation of an object is in many ways an abstracted, coded, and/or incomplete reflection of the object itself. Mental transformations are viewed as operations on the structure of a propositional network that lead to changes in the way the representation can be used.

Investigators have attempted to devise procedures that would permit inferences as to whether analog or propositional processing is being used in mental rotation tasks, despite the arguments of some that empirically based distinctions are logically impossible to make (e.g., Anderson, 1978). In fact, convincing empirical evidence that exclusively favors either propositional or analog representations has not been forthcoming.

It is likely that the requirements of the tasks required of subjects in mental representation experiments affect the apparent form of the representations used by subjects in completing those tasks. Richman, Mitchell, and Reznick (1979) have argued that, in mental scanning and travel tasks, the instructions given subjects can determine whether results consistent with an analog model or a propositional model are produced. Others (e.g., Corballis, Zbrodoff, Shetzer, \& Butler, 1978; Pylyshyn, 1979) have found that the nature of the stimulus is important in mental rotation tasks.

In fact, the requirements of the experimental task are often used by adherents of one side of the mental representation debate in criticizing research by adherents of the other side. For example, Pylyshyn (1979) designed an experiment to demonstrate that, contrary to then current theories of mental imagery, subjects "rotate" simple stimuli more rapidly than complex stimuli. 
Cooper and Podgorny's (1976) experiment, which produced results that failed to support such a complexity effect, were thus called into question. Kosslyn (1981), on the other hand, criticized the task demands of Pylyshyn's (1979) experiment, arguing that strategies were induced in subjects that resulted in data that should not be interpreted as denying the possibility of analog representation.

Although the importance of task demands is increasingly acknowledged, task demands per se have not been thoroughly investigated. Presumably, different visual comparison tasks induce different strategies in subjects, resulting in data that reflect the specific strategy that was induced. In order to determine the importance of task demands in producing specific effects, experiments are needed in which the nature of the task is changed, in a systematic way, while other factors are held constant. The present studies were designed to investigate the influence of task demands in mental representation experiments. Specifically, we investigated the extent to which the results of a typical mental rotation experiment changed as a function of the type of same-different judgment subjects were required to make.

In addition, we hypothesized that the types of patterns subjects are asked to represent and manipulate affect the apparent nature of their representation. Although the effect of figural complexity on the speed of mental rotation has been investigated often (e.g., Cooper \& Podgorny, 1976; Pylyshyn, 1979), the effect of figural organization has not. Shepard (e.g., Shepard, 1975) has referred to the impression of subjects performing his mental rotation task that the representation being rotated "breaks up," or loses its cohesiveness, if the rotation is attempted at too great a rate. Consistent with Shepard's view is the hypothesis that the representations of well organized patterns could be mentally rotated or manipulated faster, while still maintaining their cohesiveness, than could similar, but less highly organized patterns.

Human faces represent one type of pattern in which the configuration of features is important. Adults recognize patterns that are facial configurations much more rapidly than formally similar patterns composing a nonfacial configuration (e.g., Bradshaw \& Wallace, 1971), and the special nature of faces is evident even for infants, who prefer to gaze at them instead of other types of patterns (e.g., Fantz, 1961).

The perception of misoriented faces has been examined by others. Rock (1973) has noted that faces are exceedingly difficult to recognize when inverted, perhaps because the individual inverted features cannot be processed simultaneously. Carello (Note 1) attributes the difficulty of tasks requiring the recognition of inverted faces to our tendency to analyze faces in terms of invariants that do not withstand physical rotation. Unfortunately, since the mental manipulation of faces has not been experimentally compared to the manipulation of nonfaces of equivalent complexity, it is difficult to interpret findings that the recognition of inverted faces is difficult. The difficulty could result from the special nature of faces, or from the requirements imposed by a recognition task involving faces, or from some other, as yet undetermined factor.

It is, in fact, likely that the nature of the stimulus and the requirements of the task interact: Mental rotation of faces may not be possible if information necessary to carry out some types of perceptual tasks (e.g., recognition) is to be preserved. For other tasks (e.g., same-different judgments), the presence of facial structure in a stimulus may facilitate mental rotation and/or perceptual matching. Our experiments examined this hypothesis. Specifically, we hypothesized that, for our same-different matching tasks, the presence of facial organization would facilitate mental rotation: Representations of facial configurations, because of their inherent organization, should be rotated more rapidly than representations of comparable nonfacial configurations. That is, the time required to match faces should be less than that to match nonfaces, and the difference should increase with the angular difference between the configurations being compared. For other matching tasks, particularly those that discourage mental rotation, the advantage of facial organization should be less apparent.

We designed two experiments to test these hypotheses. Both experiments required subjects to make samedifferent judgments on novel, sequentially presented stimuli. Half of the stimulus pairs were scrambled, nonface versions of the original faces. In addition, the second stimulus of each pair could be rotated $0,45,90$, 135 , or $180 \mathrm{deg}$ with respect to the first. The key difference between the two experiments was in the nature of the distractors in the same-different task. In the first experiment, the "different" distractors were mirror images of the initial stimulus: the typical mental rotation task. In the second experiment, subjects were required to detect changes in the individual features of the second stimulus with regard to the first. This task was similar to that used by Cooper and Podgorny (1976), except that, in our study, mirror-image distractors were not interspersed with the changed feature distractors. Thus, if subjects use different strategies depending on the type of distractor items they are required to detect, our experiments should provide means of detecting those strategy differences.

Finally, our experiments were designed to extend the typical mental rotation task to pairs of patterns that were unfamiliar and sequentially presented. In most previous studies of mental rotation, stimuli to be rotated have consisted of either familiar objects (e.g., letters of the alphabet; Cooper \& Shepard, 1973) or objects that subjects are required to memorize in a standard orientation (Cooper \& Podgorny, 1976). Studies in which novel objects have been presented to 
subjects on every trial (e.g., Just \& Carpenter, 1976; Shepard \& Metzler, 1971) have simultaneously presented the two stimuli to be compared. We chose to extend the research on the mental rotation task by presenting novel stimuli to subjects on every trial and by presenting those stimuli sequentially.

\section{EXPERIMENT 1}

\section{Method}

Subjects. Subjects were 24 students enrolled in an introductory psychology course at the University of Minnesota. Two of the 12 female subjects were lefthanded; 1 of the 12 male subjects was left-handed.

Stimuli. The patterns used were similar to some constructed by J. J. Gibson (see E. J. Gibson, 1969, p. 106) that resembled profiles of faces (see Figure 1). There were 50 pairs of face-like patterns and 50 pairs of non-face-like patterns. In half of the pairs, the two members were identical, and in half, they were mirror images of each other.

Each facial pattern was composed of five features (hair, eyes, nose, mouth, and chin). Each feature had three different examples, except that there were seven different mouths. From this pool of 567 possible faces, 50 feature combinations were randomly chosen, with the limitation that no two faces have more than three features in common. Twenty-five randomly selected faces from this smaller pool were duplicated exactly, making up the "same" face-pattern pairs. Mirror-image "different" pattern pairs were constructed by duplicating the first pattern in each "different" pair and then reversing it so that it became a mirror image of the first. Thus, if the first slide of a "different" pair was of a face pointing toward the left, the second slide in that pair was of the identical face pointing toward the right (for 0 -deg angular disparity). For half of the pattern pairs (both face and nonface), the orientations of both members of the pair were reversed. This assured that the first pattern. of each pair conveyed information to the subject: If the pattern was pointing to the right, for example, the second patterns would be considered the same only if, allowing for any angular disparity, they, too, were pointing toward the right. This modification was necessary because without it the task would have been one of merely detecting, for example, left-facing patterns; the first patterns in each pair would not be relevant if they were all oriented in the same direction.

A second set of 50 pairs of patterns was generated by rearranging the features of each face-like pattern into a non-face-like configuration. Specifically, the "eyes," "noses," and "mouths" of the stimuli to be made into nonfaces were merely placed on the inside, rather than the outside, of the facial profiles (see
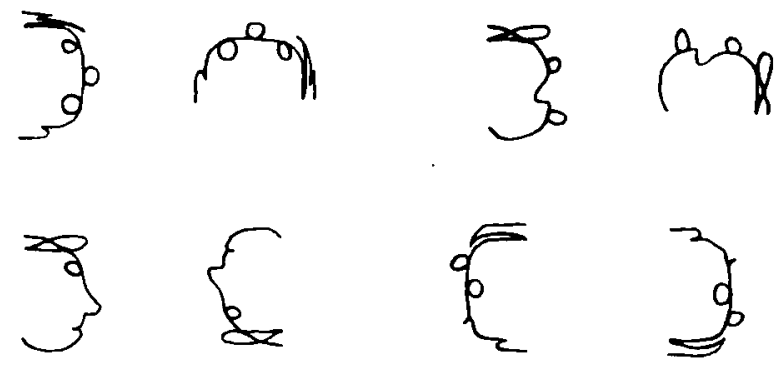

Figure 1. Examples of face and nonface patterns used in Experiment 1. At the top are "different" pairs, with the second pattern rotated $90 \mathrm{deg}$. At the bottom are "same" pairs, with the second pattern rotated $180 \mathrm{deg}$.
Figure 1). Subjects did not report being able to directly perceive the resulting stimuli as faces.

The first member of each pair was presented in an upright orientation, and the second was rotated clockwise $0,45,90$, 135 , or $180 \mathrm{deg}$. The second member was randomly assigned to one of these five possible angular orientations around a circle in the two-dimensional picture plane. The only constraint on this randomization procedure was that there be equal numbers of "same" and "different" stimulus pairs at each orientation.

Extensive pilot testing had revealed that the task was a difficult one for subjects to perform when the 100 pattern pairs were presented in a completely randomized order. Consequently, in order to reduce the error rates, the patterns were blocked by amount of rotation, as well as by face-nonface conditions. ${ }^{1}$ Thus, the stimulus set consisted of 100 pairs of patterns. They were arranged in 10 blocks of 10 pairs each: 5 "same" and 5 "different" stimulus pairs, with the second pattern of every pair in each block presented at one of five rotational angles. All of the patterns in each block were either faces or nonfaces.

Procedure. Each subject was required to judge the entire set of patterns in one $45-\mathrm{min}$ experimental session. The patterns were presented by a slide projector on a rear-projection screen. Each pattern subtended a visual angle of approximately $4 \mathrm{deg}$. Prior to the start of the first experimental sessions, subjects were shown one randomly chosen example of each of the 10 types of pattern pairs (face vs. nonface, at each rotational angle) to familiarize them with the nature of the items to be judged. The five features of each pattern were pointed out, but they were labeled ("hair," "nose," "eyes," etc.) only for faces. Subjects were also informed that there were equal numbers of "same" and "different" pairs and that the figures that were different would be mirror images of the first figure. The subject, in a given trial, viewed the first member of the pair for $7.5 \mathrm{sec}$. Approximately $750 \mathrm{msec}$ later, the second pattern of the pair replaced the first, and subjects pushed a button with their dominant hand to indicate a "same" response or a button with their nondominant hand to indicate a "different" response, to end the trial. The intertrial interval was $3.0 \mathrm{sec}$, and the interblock interval was approximately $25 \mathrm{sec}$. Reaction time was electronically recorded; the timer was started by a photocell above the projection screen and stopped by the subject's response. Trials on which errors occurred were not repeated, nor was feedback given to subjects as to the accuracy of their responses.

Following completion of the experiment, during the debriefing period, subjects were to describe the strategy they used to complete the task, in order to determine whether their introspections were consistent with those of subjects in other mental rotation experiments reported in the literature. Subjects' responses were summarized by the experimenter and recorded for later analysis.

\section{Results}

Errors. Five of the 24 subjects made more than 30 errors in the 100 trials. The mean error rate for the remainder of the subjects was $6.4 \%(\mathrm{SD}=4.5 \%)$; data from the five error-prone subjects (four female, one male) were not further analyzed. Error rates for "same" and "different" pairs of stimuli did not differ, and error rates did not depend on either face-nonface or amount of rotation conditions.

Reaction time data. The reaction time data for correct "same" responses are presented in Figure 2, and the data for correct "different" responses are presented in Figure 3. A 2 (same-different) by 2 (face-nonface) by 5 (possible rotations between 0 and $180 \mathrm{deg}$ ) repeated. 


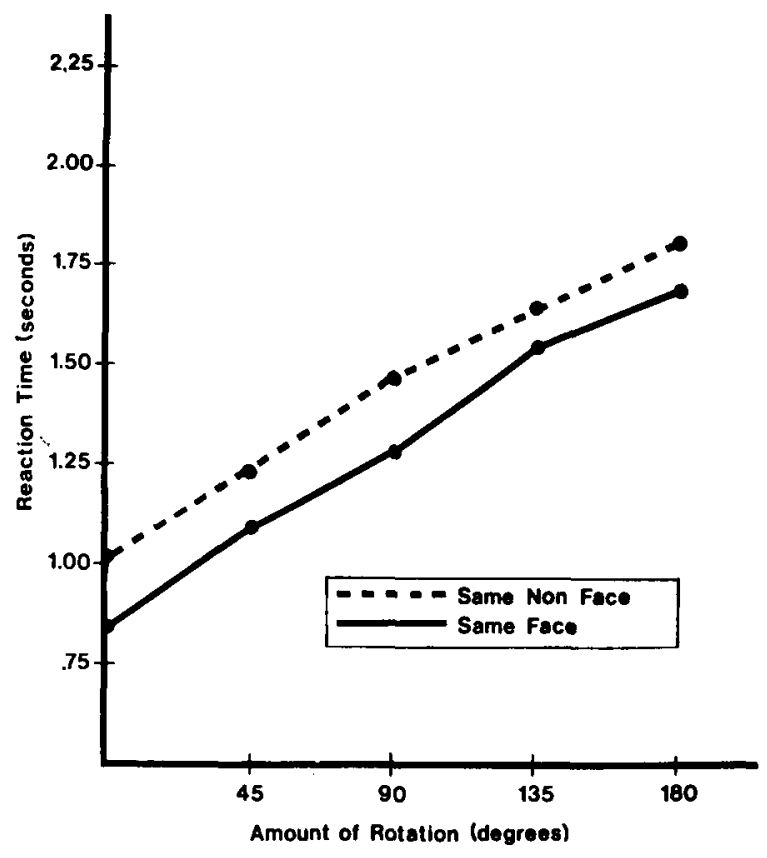

Figure 2. Mean reaction time as a function of rotation for correct "same" responses in Experiment 1.

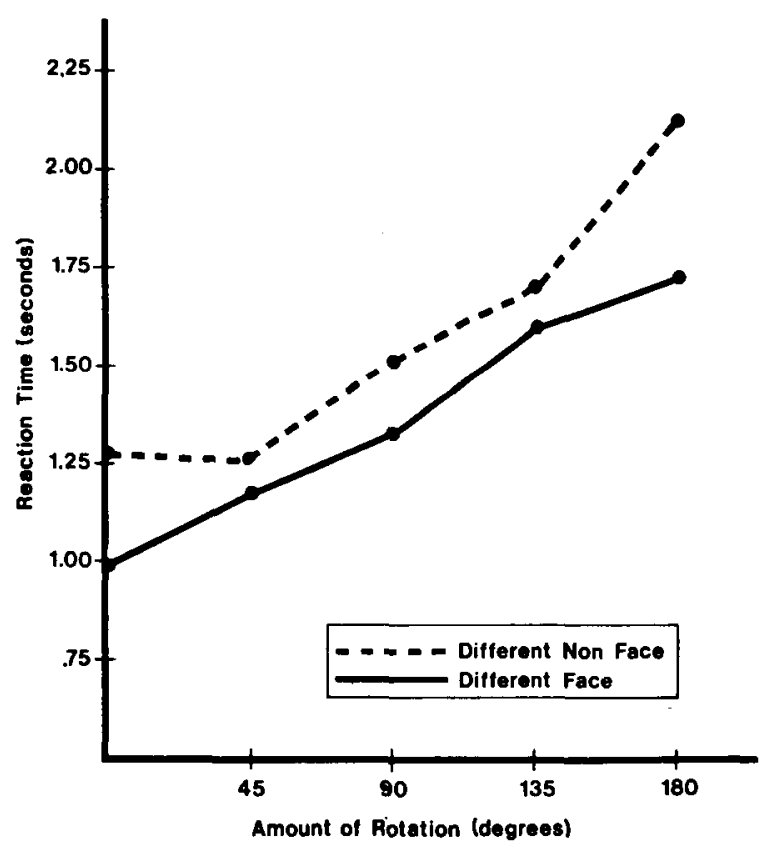

Figure 3. Mean reaction time as a function of rotation for correct "different" responses in Experiment 1.

measures analysis of variance performed on these data demonstrated significant main effects of face-nonface $[F(1,18)=10.41, p<.01]$ and of degree of rotation $[F(4,72)=12.57, p<.01]$. Although "same" judgments were faster than "different" judgments (mean "same" = $1,348 \mathrm{msec}$; mean "different" = 1,453 $\mathrm{msec}$ ), the difference did not quite reach statistical significance $[F(1,18)=3.91, p<.10]$. None of the interactions was significant (all Fs $<1.0$ ). There was a significant tendency for subjects to take longer to respond with increasing angular distance between the members of a pattern pair. However, the absence of an interaction between the amount of rotation and the facial characteristics of the pattern indicates that representations of faces were not mentally rotated or manipulated faster than those of nonfaces; rather, the differences in judgment times between faces and nonfaces were constant at each degree of angular discrepancy.

Trend analyses revealed significant linear trends over rotational position for "same" and "different" judgments of both faces and nonfaces $[5.12<F(1,90)<$ $12.73, p<.05]$, and no higher order trends were significant.

Subjects' reports. Fifteen subjects in this study reported that they performed rotation-like processes on one pattern of each pair, after concentrating on a limited number of features of the first pattern of each pair, in order to determine if the features on which they were concentrating were mirror images of each other. For example, some subjects reported that they "imagined the second slide rotating" or "turned the first picture over." The remaining four subjects could not report on their strategy. Subjects did not report examining or remembering every feature of every pattern.

\section{Discussion}

The results conform to those expected on the basis of the large literature on mental rotation. Reaction time was a linear function of the angular discrepancy between the first and second members of the pattern pairs, "same" judgments were consistently although not significantly faster than "different" judgments, and subjects' introspective accounts were similar to those previously reported in the mental rotation literature. These results were obtained despite the fact that subjects in our task were comparing novel stimuli that were sequentially presented, and thus, they provide further confirmation as to the robust nature of the mental rotation effect.

Our hypothesis that the organization provided by facial structure would facilitate mental rotation was not confirmed. There is, however, a wide variety of evidence in the literature indicating that rotation speeds vary for different types of stimuli. Letters, for example, are rotated much more rapidly than irregular polygons (e.g., Cooper \& Podgorny, 1976, vs. Cooper \& Shepard, 1973). It may be, then, that rotation efficiency in a task requiring the discrimination of mirror images is not a function of the organization of stimuli, given equivalent information content, but is, rather, a function of some other aspect of the stimulus being rotated, such as familiarity. Subjects obviously used the structure available in the facial configurations: The facial patterns were judged more quickly than were nonfacial patterns. There was no consistent evidence that subjects used different strategies in comparing facial to nonfacial patterns, but we can see that whatever strategy is used is 
facilitated by the presence of the structured facial information. The results of our first study, then, demonstrate that our stimuli and procedures can induce subjects to use strategies involving mental rotation, as described in previous literature, and that the organization of the stimuli used does not affect the speed with which that rotation is accomplished. Our second experiment was designed to determine how changes in the demands of the task affect subjects' strategies.

\section{EXPERIMENT 2}

\section{Method}

Subjects. Subjects were 49 students enrolled in an introductory psychology course at the University of Minnesota. Of the 24 male subjects, 4 were left handed. One of the 25 female subjects was left thanded.

Stimuli. The stimuli in the second experiment were identical to those used in the first experiment, except that the nature of the distractor items in "different" stimulus pairs was changed. The second pattern from each of the original "different" pairs was discarded; new changed feature distractors were constructed as follows: One of four features (any one except the eyes) was randomly selected to be changed, and its replacement was randomly selected from the remaining features of that class. (Pilot testing had indicated that changes in the eyes of the faces were too difficult to discriminate.) The stimuli in the second experiment were blocked by the amount of rotation of the second stimulus in each pair and by the face vs. nonface nature of the pair, to maintain congruence with the first experiment. Examples of the stimuli used in the second experiment appear in Figure 4.

Procedure. The procedure was identical to that used in the first study, except that each subject in the second study judged the patterns twice, $24-48 \mathrm{~h}$ apart. A second session was necessary because of the increased difficulty of the new task.

\section{Results}

Errors. Eleven of 49 subjects were essentially unable to do the task; they each made more than 40 errors in the 200 trials $(20 \%)$. These subjects' error rates were twice as high as the mean for the group as a whole. Because of the quantity of missing data for these subjects (five males, six females), the data were not further analyzed. The error data for the remaining 38 subjects are presented in Table 1 . These subjects made relatively
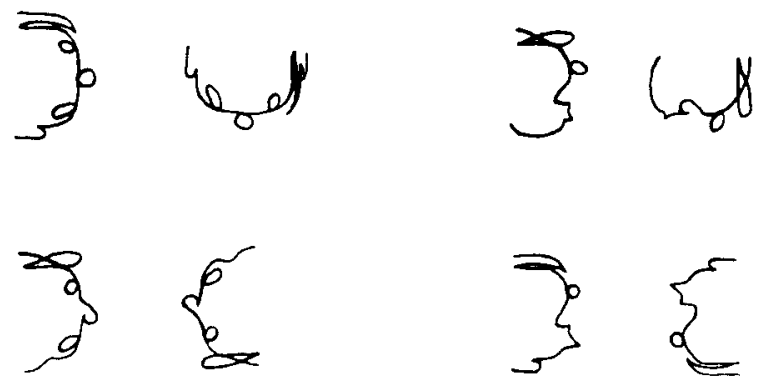

Figure 4. Examples of face and nonface patterns used in Experiment 2. At the top are "different" pairs, with the second pattern rotated $90 \mathrm{deg}$. At the bottom are "same" pairs, with the second pattern rotated $180 \mathrm{deg}$.
Table 1

Error Rates (in Percentages) for "Same" and "Different" Pairs in Two Sessions in Experiment 2

\begin{tabular}{lrrr}
\hline & Session 1 & Session 2 & Mean \\
\hline Same & 4.51 & 2.17 & 3.34 \\
Different & 23.33 & 12.07 & 17.07 \\
Mean & 13.92 & 7.12 & 10.52 \\
\hline
\end{tabular}

few errors (mean $=10.52 \%$ ), indicating that the measures taken to reduce the error rates were partially successful. It is interesting to note that, as in Cooper and Podgorny's (1976) study, the error rate for "same" pairs of patterns was uniformly lower than that for "different" pairs of patterns in both sessions [ $t(36)=$ $11.25, \mathrm{p}<.005]$. This was not the case in our first study, and it indicates that subjects often did not see feature changes when they did occur but rarely stated that a feature change had occurred when it had not. This result occurred despite the fact that subjects were told before beginning the task that there were equal numbers of "same" and "different" pairs. The error rate for faces was not significantly different from that for nonfaces for either session; but the error rate did depend on the extent to which the patterns were rotated: The larger, the rotation, the more errors subjects made.

Reaction time data. The data for correct "same" and "different" responses are presented in Figure 5 and Figure 6, respectively. To test our hypothesis that faces would be judged more quickly and that the difference would increase with degree of angular discrepancy, a 2 (replications) by 2 (same-different) by 2 (face-nonface) by 5 (rotations between 0 and $180 \mathrm{deg}$ ) repeatedmeasures analysis of variance was computed on the subjects' correct reaction times. All four main effects were significant [replications, $F(1,37)=41.67, p \ll .01$; same-different, $\mathrm{F}(1,37)=80.40, \mathrm{p} \ll .01$; face-nonface, $F(1,30)=26.07, p \ll .01$; rotational position, $F(4,148)=$ $30.64, \mathrm{p} \ll .01]$. There were no significant interactions $(.05<\mathrm{F}<2.45, \mathrm{p}>.10)$. Thus, subjects responded faster to "different" than to "same" pairs, to faces than to nonfaces, and during the second than during the first experimental session. In addition, they took longer to respond with increasing angular distance between the two patterns. However, the lack of significant interaction between the face-nonface variable and the amount of rotation reflects the fact that the overall difference between faces and nonfaces did not increase as the angular discrepancy between members of a pair increased.

On the basis of Figures 5 and 6 , the relation between the amount of rotation and reaction time does not appear to be uniformly linear, a result that is consistent with data from extensive pilot testing conducted prior to this study. A trend analysis performed on subjects' correct responses during the second, most error-free set of trials yielded significant linear trends over rotational position for both faces and nonfaces for "same" and 


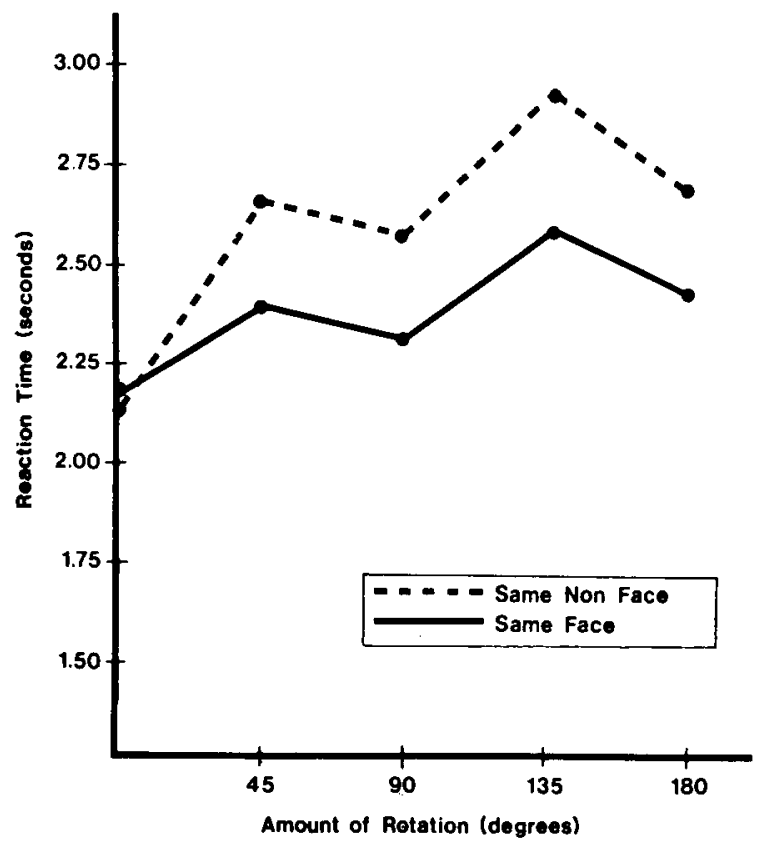

Figure 5. Mean reaction time as a function of rotation for correct "same" responses in Experiment 2.

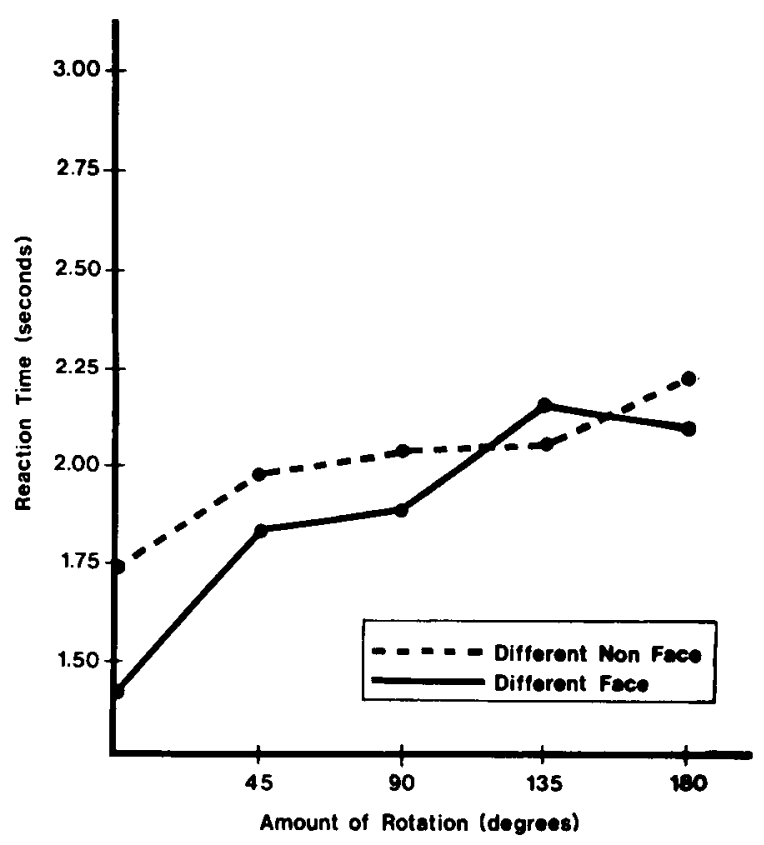

Figure 6. Mean reaction time as a function of rotation for correct "different" responses in Experiment 2.

"different" judgments $[4.86<\mathrm{F}(1,185)<15.44$, $\mathrm{p}<.05]$, and there was an additional quadratic trend for "same" judgments of nonfaces $[F(1,185)=5.92$, $\mathrm{p}<.05]$, but not for faces $[\mathrm{F}(1,185)=1.14, \mathrm{p}<.25]$. Cubic trends were not significant. Hence, while the relation between amount of rotation and reaction time for faces can statistically be best described as linear, this relation for "same" nonfaces is not strictly linear. This finding is consistent with the hypothesis that subjects' analysis of face-like patterns may be different from their analysis of non-face-like patterns. The significant quadratic trend for "same" nonfaces and the appearance of Figure 5 suggest that an oblique effect may be present in subjects' data. Oblique effects have been noted by others (e.g., Mach, 1914) in other kinds of tasks; further research is required to determine the parameters of this effect.

Subjects' reports. It was interesting that no subject reported "mentally rotating" a representation or image of the first pattern in order to compare it with the second. Rather, all subjects reported that they learned to encode the salient features of the stimuli, labeling them sequentially (e.g., from the top downward) as, for example, "round," "double looped," and so on. When presented with the second pattern, subjects reported that they then compared their remembered list of features with the presented patterns and responded accordingly.

\section{Discussion}

Despite the introspective accounts, the results obtained in this study are not completely inconsistent with those from past studies of mental rotation. Specifically, a significant relation was found between the angular disparity between two patterns and the amount of time required by subjects to judge whether they were the same. This relation was found despite the fact that, unlike most previous experiments, the patterns were relatively unfamiliar to the subjects, were presented sequentially rather than simultaneously, and were to be discriminated solely from changed feature distractors. However, these results are very different from those of Experiment 1, in which reaction time was a linear function of angular discrepancy for faces and for nonfaces, and in which "same" reaction times tended to be faster than "different" reaction times at every orientation.

A comparison of reaction times for the correct "same" responses in the two studies is particularly enlightening. The subjects in these two conditions were judging identical pairs of patterns. The "same" pattern pairs were identical in every respect in the two studies. Nevertheless, a comparison of Figures 2 and 5 demonstrates that the processing of those identical stimuli required a full $1 \mathrm{sec}$ longer, on average, in Experiment 2 than in Experiment 1. This difference between the two experiments was considerably larger than any other effect. There was also a difference in the slope of the reaction time functions in the two experiments. The failure of our second experiment to replicate the consistent findings of studies of mental rotation is especially interesting in light of our first experiment, in which the expected results were obtained. The crucial difference between the two studies, of course, was in the criteria by which subjects judged the similarity of members of the pattern pairs. Mental rotation strategies seem to be very efficient, as the data from Experiment 1 indicate, and subjects may well use those strategies, when possible. In Experiment 2, however, subjects had to attend to all 
of the features of both patterns in each pair, which may have prevented or discouraged their using the same strategy used by subjects in the first study.

The most economical explanation for the data in the second experiment may be that reflected in the subjects' introspective accounts that the features of each pattern are encoded as a list that is then compared to the subsequent pattern feature by feature. If one assumes that features that are rotated can nevertheless be recognized, the effect of angular disparity on reaction time would be accounted for if, the farther from the original location the first feature on a subject's list appears, the more difficult it is to locate. The greater the rotation, the greater the displacement of that first feature and, hence, the longer the subject takes to judge the pattern pair. Once the starting point of the feature list is located, the rate at which the features are located and compared might depend in part on organizational characteristics of the stimulus. Since the features of faces and nonfaces were organized in the same way, the difference in reaction time between faces and nonfaces should not be due to subjects' ordering their lists of features differently. Rather, the features of face-like stimuli may be more readily identified and more readily located, whereas features of the nonface stimuli may be more difficult to identify and require longer periods of time to locate. Hence, once the subjects begin to process their feature lists, they do so more rapidly for faces than for nonfaces.

Of course, this hypothesis is post hoc, but it receives some support from the mean reaction times for "same" vs. "different" responses: Whereas in all previous mental image rotation tasks in which mirror images were used as distractors, "same" responses were faster than "different" responses (e.g., Cooper \& Shepard, 1973), in the present study, they averaged $500 \mathrm{msec}$ slower. Although there were fewer errors on "same" than on "different" judgments, it is unlikely that slower reaction times for "same" judgments could be due to subjects' foregoing speed for accuracy, since (1) subjects could not know, before their decision was made, whether an item would be the same or different and (2) the error rate for faces was not different from that for nonfaces, despite different reaction times for that manipulation. The reaction time difference between "same" and "different" stimuli is consistent with a strategy of scanning a list of features and responding as soon as a "different" feature is noticed. On the average, only two features would have been compared at this point, whereas all four features would need to be compared to determine that a pattern is identical.

In summary, the second study yielded some results that were unexpected in the context of our first experiment and previous mental rotation research. The constant difference between subjects' judgment times for the two types of patterns, the subjects' own introspective accounts, and the fact that "different" judgments were faster than "same" judgments suggest that the strategies used by subjects in Experiment 2 may have been different from those typically used in performing the usual mental rotation tasks. In particular, the requirement of the present task that subjects detect changes in the features of patterns may have led them to use strategies different from those used to detect mirror images of patterns.

It is, of course, possible that mental rotation was carried out before feature comparison began in both experiments and that serial feature comparison was then carried out by subjects in Experiment 2 and holistic comparison was carried out by subjects in Experiment 1 . This two-stage account is not well supported by the data; in particular, the difference in slopes of the reaction time functions in Experiment 1 and Experiment 2 would appear to indicate that stimuli in Experiment 1 were more rapidly "rotated" than those in Experiment 2 even though the stimuli themselves were physically identical.

It is also conceivable that the nature of a matching task could affect the nature of mental rotation, but such an account requires the concept of mental rotation to be much more complex and is not as plausible as an account in which "mental rotation," as discussed by other investigators, is used in some situations, and strategies not involving mental rotation are used in other situations. One implication of this hypothesis is that the nature of the task itself may determine the use of rotation-like strategies by subjects, and we know virtually nothing about situations in which each strategy may be preferred.

Cooper and Podgorny (1976) discovered consistent individual differences in a mental rotation task that strikingly parallel the differences we found between our two tasks. Specifically, Cooper and Podgorny found that one type of subject (Type 1) produced "same" responses more rapidly than "different" responses and produced both types of responses much more rapidly than Type 2 subjects did. Type 2 subjects produced "different" responses more rapidly than "same" responses. Cooper and Podgorny described Type 1 subjects as using a holistic, rapid comparison process, whereas Type 2 subjects were thought to use more analytic, feature-byfeature comparison strategies. As mentioned earlier, Cooper and Podgorny intermingled mirror-image distractors with changed-feature distractors; subjects presumably chose the strategy that they were able to use most effectively overall.

The results of our second experiment parallel those characteristics of Cooper and Podgomy (1976) Type 2 subjects: "Different" responses were more rapid than "same" responses, but both types of responses required more time to produce than did those of subjects in our first experiment. It may be that our different procedures induced subjects to use different strategies; Cooper and Podgorny's procedure may have encouraged subjects to select one strategy for both kinds of distractors.

The fact that subjects judging highly similar patterns 
produced highly dissimilar reaction time functions could indicate that the debate about the nature of mental representation (propositional vs. analog), at least insofar as it depends upon evidence from studies of mental rotation, could be better aimed at determining the circumstances under which subjects use different strategies rather than at attempting to conclude that they use one strategy or another. It is reasonable to conclude from our studies that subjects use different strategies depending upon the nature of the comparisons they are required to make. Recent theories of cognitive performance have explicitly acknowledged the effects of task demands and other aspects of the context in which an experiment is conducted on the results derived from such experiments (e.g., Jenkins, 1977). Our studies present convincing empirical support for such theories. Subjects, given identical stimuli on which to make "same" judgments, engage in vastly different strategies, depending on the nature of accompanying "different" judgments.

In summary, these studies have demonstrated both generality and specificity of the strategies subjects use in tasks in which they are required to compare patterns and decide as quickly as possible whether they are identical. When the patterns may differ by being mirror images of each other, subjects can use strategies like those used in other mental rotation tasks even though, in the present case, the patterns were unfamiliar and sequentially presented. On the other hand, when the members of a pair may differ by a feature, subjects apparently use quite a different strategy for making the comparison, one that requires considerably more time and that may involve systematically scanning the patterns feature by feature.

\section{REFERENCE NOTE}

1. Carello, C. On faces. Paper presented at the Eastern Ecological Psychology Society, Hartford, September 1981.

\section{REFERENCES}

Anderson, J. R. Arguments concerning representations for mental imagery. Psychological Review, 1978, 85, 249-277.

Bradshaw, J., \& Wallace, G. Models for the processing and identification of faces. Perception \& Psychophysics, 1971, 9, 443-448.

Cooper, L. A., \& Podgorny, P. Mental transformations and visual comparison processes: Effects of complexity and similarity. Journal of Experimental Psychology: Human Perception and Performance, 1976, 2, 503-514.

Coopen, L. A., \& She Pand, R. N. Chronometric studies of the rotation of mental images. In W. O. Chase (Ed.), Visual information processing. New York: Academic Press, 1973.
Corballis, M., Zbrodoff, N., Shetzer, L., \& Butler, P. Decisions about identity and orientations of rotated letters and digits. Memory \& Cognition, 1978, 6, 98-107.

F ANTZ, R. L. The origins of form perception. Scientific American, 1961, 204, 66-72.

Gibson, E. J. Principles of perceptual learning and development. Englewood Cliffs, N.J: Prentice-Hall, 1969.

Jenkins, J. J. Remember that old theory of memory? Well, forget itl In R. Shaw \& J. Bransford (Eds.), Perceiving, acting, and knowing. Hillsdale, N.J: Erlbaum, 1977.

Just, M. A., \& Carpenter, P. A. Eye fixations and cognitive processes. Cognitive Psychology, 1976, 8, 441-480.

KossLyn, S. M. The medium and the message is mental imagery: A theory. Psychological Review, 1981, 88, 46-68.

Kosglyn, S. M., \& Pomerantz, J. R. Imagery, propositions, and the form of internal representations. Cognitive Psychology, 1977,9, 52-76.

МACH, E. [The analysis of sensations] (S. Waterlow, trans.). Chicago: Open Court, 1914.

Palmer, S. E. Visual perception and world knowledge: Notes on a model of sensory-cognitive interaction. In D. A. Norman \& D. E. Rumelhart (Eds.), Explorations in cognition. San Francisco: Freeman, 1975.

Pylyshy, $Z$. W. What the mind's eye tells the mind's brain: A critique of mental imagery. Psychological Bulletin, 1973, 80, $1-24$.

PyLYshyn, Z. W. Validating computational models: A critique of Anderson's indeterminacy of representation claim. Psychological Review, 1978, 86, 376-382.

Pylyshyn, Z. W. The rate of "mental rotation" of images: A test of a holistic analog hypothesis. Memory \& Cognition, 1979, 7, 19-28.

Pylyshyn, Z. W. The imagery debate: Analogue media versus tacit knowledge. Psychological Review, 1981, 88, 16-45.

Richman, C. L., Mitcheld, D. B., \& Reznick, J. S. Mental travel: Some reservations. Journal of Experimental Psychology: Human Perception and Performance, 1979, 5, 13-18.

Rock, I. Orientation and form. New York: Academic Press, 1973.

SHEPARD, R. N. Form, formation, and transformation of internal representations. In R. Solso (Ed.), Information processing and cognition: The Loyola Symposium. Hillsdale, N.J: Erlbaum, 1975.

She PAnd, R. N. The mental image. American Psychologist, 1978, 33, 125-137.

Shepard, R. N., \& Metzler, J. Mental rotation of threedimensional objects. Science, 1971, 171, 701-703.

\section{NOTE}

1. Although blocking by amount of rotation has been shown to affect subjects' response times (e.g., Cooper \& Shepard, 1973), this is true only when subjects are aware of the stimulus to be presented. Since our subjects did not know, before the stimuli were presented, what the stimuli would actually look like, they were not able to make use of the information provided by blocking in any specific way.

(Received for publication November 24, 1981; revision accepted September 17, 1982.) 